\title{
Actitudes y prácticas respecto al uso de las redes sociales por parte de estudiantes de Educación Secundaria
}

Melissa Yudelka Pérez Falcón melivip10@gmail.com

Tutor: MSc. Franklin J. Solís Zúniga franksolis23@yahoo.com

Facultad Regional Multidisciplinaria de Estelí, Carrera Trabajo Social

\section{RESUMEN}

La investigación está orientada al uso de las redes sociales por parte de los estudiantes de décimo año C del Instituto Nacional Reino de Suecia. El objetivo era promover, conjuntamente con la comunidad educativa, un cambio de actitud y prácticas respecto al uso de las redes sociales en los estudiantes Este estudio se realizó a partir de un enfoque cualitativo, desde la perspectiva de Investigación Acción Participativa, perteneciente al paradigma sociocrítico. Las técnicas utilizadas para la recolección de datos fueron: la encuesta, entrevistas a profundidad y grupo focal. La información obtenida se analizó en función de los objetivos formulados y según su naturaleza cualitativa. Los resultados del diagnóstico reflejaron que los han tenido problemas en las redes sociales debido al uso irresponsable de estas, que han sido víctimas de ciberbullying y que las consecuencias de esto han transcendido de lo virtual a lo personal. Los docentes, respecto a este problema, expresaron que el rendimiento académico se ve afectado al dedicar mucho tiempo a las redes sociales y sugirieron actividades para mejorar el uso de las redes sociales. Como resultado principal de la investigación se logró promover un cambio de actitudes y prácticas respecto al uso de las redes sociales a partir de la experiencias vividas, así como la proposición de soluciones por parte de los estudiantes para mejorar el uso de las redes sociales, la creación de un página educativa en la red social facebook haciendo énfasis en el ciberbullying y el involucramiento de los docentes en cada una de las actividades. Por tal motivo se puede decir que se comprobó la hipótesis de acción planteada.

Palabras clave: Actitudes, prácticas, estudiantes, educación secundaria, redes sociales, investigación acción participativa.

\section{SUMMARY}

The research is oriented to the use of social networks by sophomores year C of the Kingdom of Sweden National Institute. The aim was to promote, together with the educational community, a change of attitude and practice regarding the use of social networks in students. This study was conducted from a qualitative approach, from the perspective of Participatory Action Research, part of the paradigm sociocritic. The techniques used for data collection were: survey depth interviews and focus group. The information obtained was analyzed in terms of the stated objectives and according to their qualitative nature. Diagnostic results reflected that have had problems in social networks due to the irresponsible use of these, which have been victims of cyberbullying and the consequences of this have transcended from the virtual to the personal. Teachers, on this issue, said that academic performance is affected by dedicating much time on social networks and suggested activities to improve the use of social networks. The main result of the investigation was possible due to promote a change of attitudes and practices regarding the use of social networks from life experiences, and proposing solutions by students to improve the use of social networks, creating an educational website on the social network facebook emphasizing cyberbullying and involving teachers in each of the activities. Therefore we can say that the action raised hypothesis was verified.

Keywords: Attitudes, practice, students, secondary education, social networks, participatory action research 


\section{INTRODUCCIÓN}

Según Ureña (2011) las redes sociales se han convertido en un medio de comunicación masivo en nuestro país, estas pueden puede utilizarse para diversos fines de acuerdo a los propósitos que tenga la/el usuario, las actividades más habituales que realizan las y los usuarios de redes sociales cuando se encuentran conectados a ellas: comunicarse con amigos, compartir información (fotos, videos, etc.) comunicarse con familiares, consultar información sobre diversos temas.

Así como existen ventajas de hacer uso de las redes sociales, también en estas se puede generar violencia hacia las y los adolescentes, este es el caso de ciberbullying en el cual se desarrollan conductas hostiles, se ataca a la reputación, daña la intimidad a través de comentarios, se inventan historias, se crean perfiles falsos, suplantación de personalidad, etiquetan fotos, insultan, chantajean con subir fotografías, extorsiones, amenazas o acosos que son los principales riesgos que pueden correr un usuario de redes sociales. Herrero, (2010)

En el caso de las y los estudiantes al hacer uso de las redes sociales sin planificación se pierde la noción del tiempo. Extender el tiempo de conexión hasta horas de la madrugada no es recomendable puesto que habituarse a estas termina pasándole la factura al organismo e incide en el bajo rendimiento académico del estudiante. El estudiante llega a clase desganado, malhumorado, somnoliento y sin poder prestar atención a las explicaciones del maestro, debido a que pierden horas de sueño por dedicar tantas horas a sus redes sociales y el organismo no recupera las energías gastadas esto da lugar al bajo rendimiento académico. Martínez, (2012).

Por las razones antes mencionadas es que surge la necesidad de promover conjuntamente con la comunidad educativa un cambio de actitudes y prácticas respecto al uso de las redes sociales en estudiantes. El grupo seleccionado está formado por 45 estudiantes pertenecientes a décimo C del Instituto Nacional Reino de Suecia de la ciudad de Estelí, debido a que en la realización del diagnóstico se identificaron problemáticas ocasionadas por el uso irresponsable de las redes sociales y a partir de estas se seleccionaron las actividades del plan de acción ejecutado.

Finalmente, conviene aclarar que la parte práctica de la investigación gira en torno a los objetivos de actuación, para ello inicialmente se partió de la elaboración de un diagnóstico y ejecución de un plan de acción de donde se derivan los resultados obtenidos con la experiencia 


\section{MATERIAL Y MÉTODOS}

Esta experiencia corresponde al enfoque de Investigación Cualitativa, siendo su carácter el de Investigación Acción Participación, la cual según Kemmis y McTaggart (1998), es una forma de indagación introspectiva emprendida por participantes en situaciones sociales o educativas, así como la comprensión de esas prácticas y de las situaciones en que estas tienen lugar. Esta se llevó a cabo a través de un espiral de ciclos de investigación y acción constituidos en las siguientes fases: planificación, actuación, observación y reflexión, se inició con la identificación de una necesidad a través de un análisis, posteriormente se elaboró y ejecutó un plan de acción y finalmente se evaluaron los resultados obtenidos. En ella participaron 45 estudiantes de décimo año del Instituto Nacional Reino de Suecia, cinco docentes que imparten clases al grupo, así como subdirectora y consejera escolar del turno vespertino.

Previamente a la elaboración del plan de acción, se realizó un diagnóstico para indagar acerca del uso que las y los estudiantes dan a las redes sociales, mediante la aplicación de una encuesta, una entrevista y un grupo focal dirigidos a estudiantes y docentes. Luego de haberse interpretado la información se identificaron las problemáticas y se procedió a la selección de ocho actividades que conformaron el Plan de Acción y en cuya ejecución participaron las y los estudiantes, así como docentes, posteriormente se procedió a la evaluación de la incidencia del plan de acción en las y los participantes. La información obtenida se analizó en función de los objetivos formulados, fue organizada en matrices de doble entrada y representada gráficamente

\section{RESULTADOS}

\section{Resultados de la etapa diagnóstica}

Objetivo: Indagar el uso que las y los estudiantes de décimo año $C$ dan a las redes sociales.

\section{Encuesta}

El 50\% de las y los estudiantes hace uso de la red social Facebook, el $28 \%$ de Youtube, el $12 \%$ de Twitter y un $10 \%$ de Ask. Fm, motivo por el cual la página "Hace oír tu voz, denten el ciberbullying" fue elaborada en la red más utilizada, Facebook. 


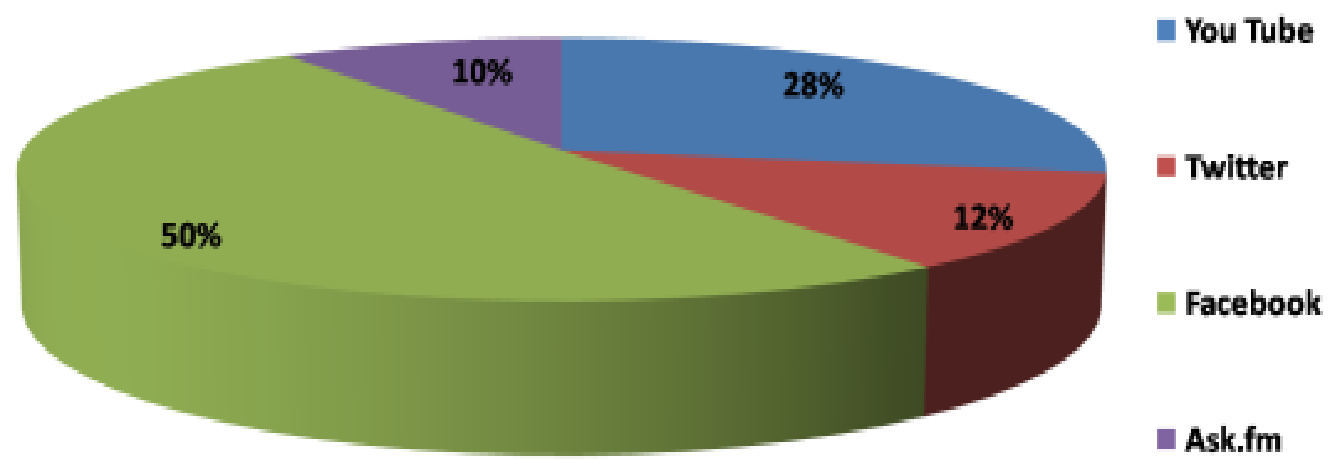

Figura 1. Redes sociales que más emplean.

Un dato encontrado y muy preocupante es el hecho de que el $91 \%$ piensa que es más fácil establecer comunicación a través de las redes sociales que de manera personal, lo que implica el distanciamiento de sus amistades y familiares reales, de esta manera la comunicación con sus allegados/as se ven afectadas al permanecer conectados y fortalecer una relación virtual.

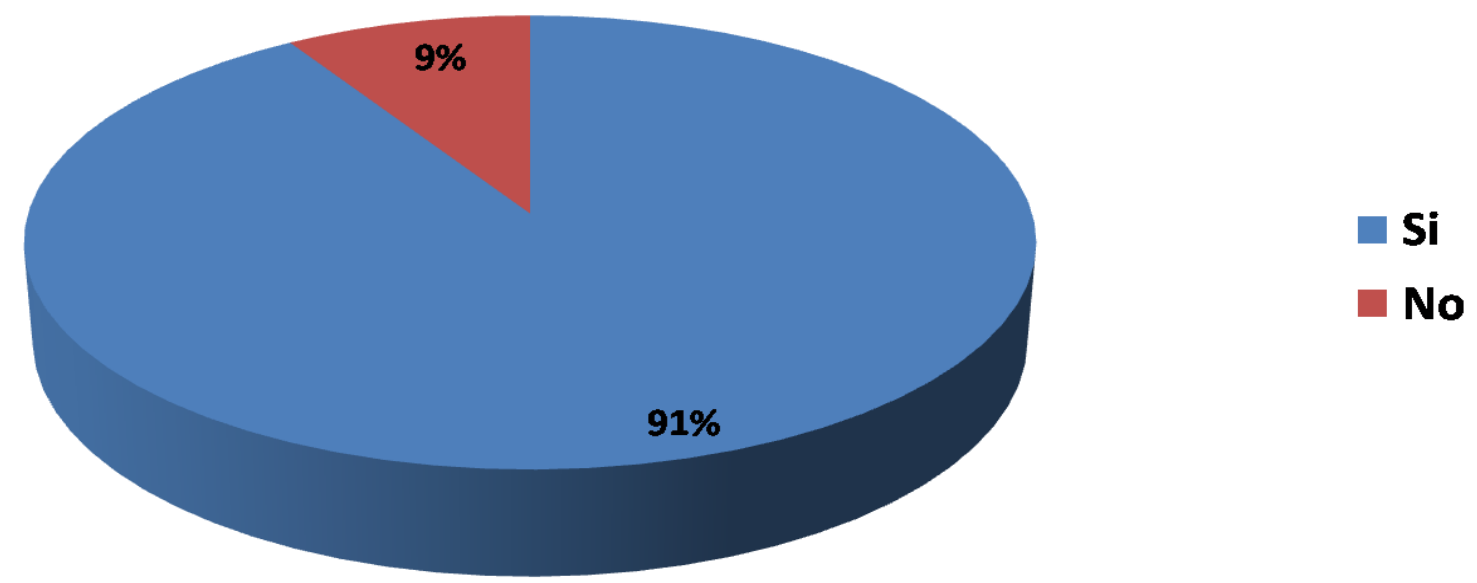

Figura 2. Opinión sobre si es más fácil la comunicación virtual 


\section{Entrevista a estudiantes}

Posteriormente a la realización de las encuestas, se seleccionaron a tres estudiantes quienes participaron en las entrevistas a profundidad. Encontrándose que las y los estudiantes han hecho uso de las redes sociales partir de los 11 y 13 años y expresaron que las redes sociales que más conocen son Facebook, Youtube, Instagram, Twitter y Ask.FM, debido a que son fáciles de usar y tienen aplicaciones que les llaman la atención. Otro problema encontrado es que han sido víctimas de burlas en las redes sociales mediante comentarios en fotografías que sus amigos suben con mala intención, es decir, ciberbullying, no obstante, no tenían un concepto claro de este término, al final indicaron que la manera de acabar con estos problemas es eliminando la cuenta en las redes sociales o bloqueando a la persona que está ocasionando daño en las redes sociales.

Las adolescentes entrevistadas también expresaron que una de las problemáticas que han sufrido en facebook es que adultos se han hecho pasar por adolescentes de su edad y por esto han sido amenazadas por sus compañeras de vida. A continuación, el relato de una de las estudiantes entrevistada:

Yo una vez me mandó la solicitud un señor un muchacho yo no lo conocía entonces le acepte la solicitud empezamos a chatear y me empezaba a decir cosas como enamorándome timándome y entonces yo le contestaba normal y entonces en una de tantas parece que la mujer de él, tenía una mujer y tenía la contraseña y se armó un problemón y hasta me trataron de todo.

\section{Entrevista a docentes}

En la entrevista realizada a docentes que imparten clases a décimo "C" se logró percibir que consideran que el uso de las redes sociales afecta a las y los estudiantes en lo que respecta al rendimiento académico, debido al uso de las redes sociales en horas clases e incumplimiento de tareas en casa.

Entre las medidas que aplican en caso de uso de celular para revisar redes sociales en horas clases es hacer llamados de atención a sus estudiantes, aplicación del reglamento, es decir, expulsión de 3 a 15 días y en ocasiones se restringe el uso de celular hasta que los padres de familia asisten a una reunión por tal comportamiento.

Alternativas que propusieron ante este comportamiento:

"Buscar la forma para que las y los estudiantes utilicen las redes sociales para cuestiones académicas". 
"Realización de charlas sobre beneficios y perjuicios".

"Invitación a expertos en este tema para que impartan a los estudiantes seminarios".

"Orientación y consejería de los padres de familia a sus hijos en lo que respecta al uso de las redes sociales".

\section{Grupo focal}

En el grupo focal se hizo énfasis en el ciberbullying que las y los estudiantes han recibido en las redes sociales, se logró reafirmar que han sufrido este fenómeno a través de comparaciones para saber quién es más bonita/o, comentarios ofensivos en fotografías y que las y los compañera/os de clases han sido agresores, esto ha afectado su autoestima, debido a que se les hace penoso salir a enfrentar los comentarios de manera personal. Las adolescentes se han sentido "feas" al no ganar en los versus, ante esto lo que les queda es tomar la situación con "calma" siguiendo el juego.

"A mí me ponen en eso de los versus y no me gusta porque una vez yo perdí y después me andaban molestando los chavalos diciendo que soy fea porque no gané en el versus."

\section{Hipótesis de acción}

En base a los resultados del diagnóstico, se planteó la siguiente hipótesis de acción:

Al impartir charlas, crear una página educativa en facebook y realizar actividades en las que las/os estudiantes puedan integrarse en conjunto con la comunidad educativa del centro, se logrará contribuir a un cambio de actitudes con respecto al uso de las redes sociales.

\section{RESULTADOS DEL PLAN DE ACCIÓN}

Uno de los resultados del plan de acción es la creación de una página en la red social facebook llamada "Hace oír tu voz, detené el ciberbullying-HODC", en la cual se publican fotografías relacionadas con el uso adecuado a las redes sociales y recomendaciones para hacer frente al ciberbullying, hasta el momento tiene 107 me gusta. 


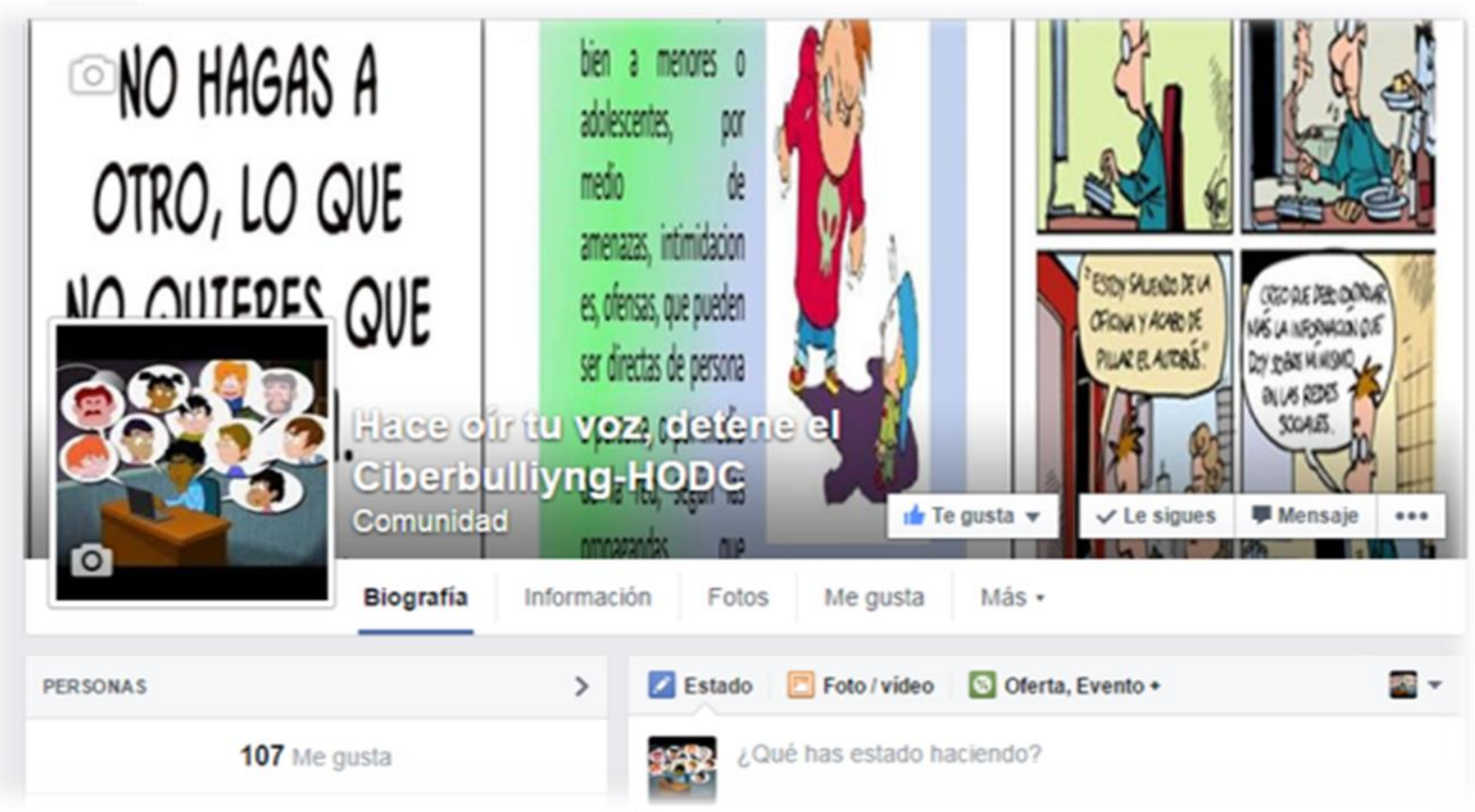

Se proporcionaron herramientas y se facilitaron medidas para que las y los estudiantes hagan uso responsable de las redes sociales, se logró hacer conciencia en lo que respecta a los peligros existentes en las redes sociales, se brindaron espacios para que compartieran sus experiencias positivas y negativas, de igual manera entre ellas y ellos se proporcionaron posibles soluciones ante el problema de ciberbullying. A continuación alguna expresiones de los estudiantes:

"Me sentí muy bien porque podemos poner en práctica lo que son las redes sociales, en facebook, celulares, correo electrónico, Youtube etc. Tenemos que tener más cuidado aprender a usarlos como un medio de comunicación y no como un medio de violación."

"Fue una experiencia muy buena ya que hicimos un mural de las redes sociales ya que es un tema muy bueno ya que todos los adolescentes están involucrados en las redes sociales pero están recibiendo bullying a través de internet, muy buena la experiencia."

A través de la realización del plan de acción se respondió a la pregunta y objetivo central de la investigación, ya que las y los estudiantes al final de la Investigación Acción Participativa, mostraron el cambio a través de su manera de actuar en la red social Facebook e impartieron lo aprendido a otros estudiantes del Instituto Nacional Reino de Suecia, otro punto importante es que apreciaron otros peligros a los que están expuestos al chatear con desconocidos durante todo el día, también vieron las 
consecuencias del uso desmedido de las redes sociales, como el engaño y el abuso sexual, vieron como la familia se ve afectada cuando el uso irresponsable de las redes sociales ocasiona consecuencias graves.

\section{CONCLUSIONES Y COMPROMISOS}

Con la ejecución del plan de acción, se logró promover conjuntamente con la comunidad educativa al cambio de actitudes y prácticas respecto al uso de las redes sociales, en estudiantes de décimo año C del Instituto Nacional Reino de Suecia, ya que las y los docentes, director y subdirectora estuvieron presentes en el desarrollo del Plan de Acción, facilitaron el acceso al grupo y brindaron su aporte para poder incidir en el cambio.

La mejor forma de sensibilización hacia las y los estudiantes fue haciendo ver a través de las charlas y consejería que los problemas que han tenido en las redes sociales se deben al mal uso que les dan y fueron ellas y ellos mismos quienes proporcionaron las soluciones y de eso se trata el plan de acción, de llevar a cabo las soluciones que las y los participantes proponen para el cambio de sus situación, de esta forma se brindó les acompañamiento, debido a que no se trató de llevar soluciones concretas, sino de tomar en cuenta sus propuestas para mejorar el uso de las redes sociales.

Por lo tanto, se puede decir que se comprobó la hipótesis de acción.

"Fue una experiencia muy buena ya que hicimos un mural de las redes sociales ya que es un tema muy bueno ya que todos los adolescentes están involucrados en las redes sociales pero están recibiendo bullying a través de internet, muy buena la experiencia."

\section{Compromisos para mejorar la acción}

Para seguir avanzando en este proceso y en base a las dificultades surgidas durante el mismo, se considera necesario:

- Continuar administrando la página en Facebook “Hace oír tu voz, detene el ciberbullying” conseguir más me gusta, publicar mensajes para mejorar el uso de las redes sociales y seguir haciendo énfasis en el ciberbullying.

- Conseguir el involucramiento de los padres de familia, ya que por cuestiones de tiempo no se logró en el presente plan de acción.

- Realizar más actividades y alcanzar otros grupos de estudiantes para contribuir en el buen uso de las redes sociales. 
- Aprovechar al máximo las energías de las y los estudiantes para que de esta forma no dejen de ser ellos mismos.

\section{REFERENCIAS}

Hernández. R, Fernández. C y Baptista. M. (2007) Metodología de la investigación. (4ta.ed) Mexico: McGRAW-HILL/ Interamericana. Editores.S.A.De.Cv.

Herrero, M. L. (2010). Redes sociales de internet y adolescentes. http://www.websatafi.com. Obtenido de http://www.websatafi.com/joomla/attachments/125_REDES\%20SOCIALES\%20PUBLICAR.pdf

Kemmis, S. y McTaggart, R. (1988). Cómo planificar la investigación acción. Barcelona: Alertes.

Martinez, A. L. (2012). ¿Cómo afectan las redes sociales el desempeño académico en adolescentes. http://www.slideshare.net Recuperado el 30 de Mayo de 2013, de http://www.slideshare.net/savedfiles?s_title=lucero-ensayo-como-afectan-las-redes-sociales-en$\underline{\text { el-desempeo-acadmico\&user_login=luceronegma }}$

Ureña, A. F. (2011). Las redes sociales en internet. www.ontsi.red.Recuperado el 12 de Mayo de 2013, de www.ontsi.red.esontsititesdefaultifilesredes_sociales-documento_0.pdf 